\title{
Dipole-vortex structure of the obscuring tori in active galactic nuclei
}

\author{
Elena Yu. Bannikova and Victor M. Kontorovich \\ Karazin Kharkov National University, 4 Svobody Sqr., 61077 Kharkov, Ukraine \\ and \\ Institute of Radio Astronomy of the National Academy of Science of Ukraine, \\ 4 Chervonopraporna Str., 61002 Kharkov, Ukraine \\ email: bannikova@astron.kharkov.ua,vkont@ira.kharov.ua
}

\begin{abstract}
A "matrjoshka" scheme for obscuring vortex torus structure is proposed, which could help to explain the AGN radiation flares, variability and evolution.
\end{abstract}

Keywords. Galaxies: active - instabilities

\section{Introduction}

Starting with the Antonucci and Miller's outstanding work, tori have been considered as a necessary element of the AGN-structures forming the basis of the AGN unified model. A brilliant achievement was the first direct observation of the obscuring tori described by Jaffe, Meisenheimer, Röttgering et al. (2004). Existence of tori was confirmed by observation with VLT optical interferometer equipped with MIDI IR-camera.

We propose to consider a torus as a dynamic object with its proper vortex motion. It is well known that two independent rotation types are possible in a torus: "orbital" - along the torus big circle (with radius $R$ ), and "vortical" - along its small circle (with radius $r$ ). This latter will be of our major interest. Vortical motion in a self-gravitating torus

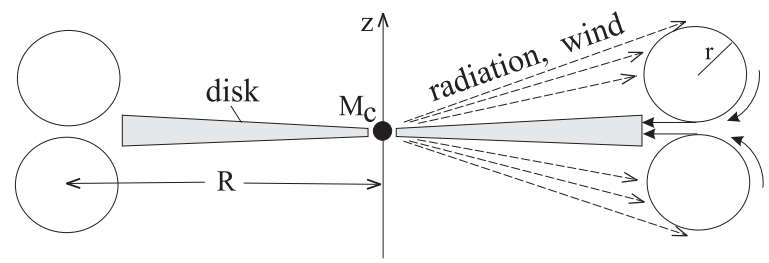

Figure 1. Proposed scheme of the dipol-toroidal vortex in the center of AGN: section by symmetry plane ortogonal to disk; $z$-axis of symmetry. Arrows show the possible matter movement.

(see discussion in Bliokh \& Kontorovich 2003) principally differs from pure orbital one. For the near Eddington luminosity $L \approx L_{\mathrm{Edd}}$, when the gravitation is largely balanced by light pressure, this motion in AGN is not so noticeable and can be quite neglected at first. Originating from or being sustained by the central source radiation or wind "twist", the vortical torus motion is capable of "replenishing" the accretion disk mass, thereby adjusting the process of accretion and introducing feedback (Fig. 1; see Bannikova $\&$ Kontorovich 2006). Here the dipole structure of a toroidal vortex, defined by the symmetry of radial-outflowing wind and radiation, is important. Note that the structure of the lines of flow across such dipole vortex resembles the structure and topology of streamlines in the well-studied hydrodynamic models, such as Hill and Lamb's vortices. 
At the same time, each component of a toroidal dipole, taken separately, resembles the Maxwell vortex, but with the opposite direction of rotation.

\section{Discussion}

The aforesaid proves that positive feedback and related instability with linear increment $\alpha=\xi \xi_{1} \zeta c /(2 R)$ are possible in the considered system. Here $c$ is light velocity, $R$ is the torus big radius, $\xi \sim 0.1$ means the efficiency of conversion of accretion energy into radiation, $\xi_{1}$ is a portion of small torus radius characterizing the matter inflow from torus to disk, factor $\zeta$ takes into account the effect of torus shape, absorption and angular dependence of radiant flux. Note that the time delay $\tau$ in a feedback circuit of accretionwind instability reduces the increment as $\alpha \rightarrow \alpha f(\alpha \tau)$, where universal function $f<1$. The accretion-wind instability increment determines the characteristic time scale of its development. For the 3.5-year time scale of the observable burst duration in quasars (see Babadzhanyants \& Belokon 1985) and $\xi_{1} \sim \zeta \sim 0.1$ we should take the space scale $R \sim 10^{16} \mathrm{~cm}$.

Since the preliminary observational data Jaffe, Meisenheimer, Röttgering et al. (2004) point at significantly larger torus sizes, it should be natural to suggest the "matrjoshka" scheme: there are tori of smaller radii within the outer big torus. In the case of Eddington luminosity, the mass of torus that replenishes the accretion disk is proportional to its big radius. In particular, for $R \sim 10^{16} \mathrm{~cm}$, the mass of central black hole $10^{9} M_{\odot}$ and accepted above parameters - the mass of inner torus $M_{\text {torus }} \sim 10^{3} M_{\odot}$. The inner toroidal vortex may be responsible for the variability of AGN, the development of instability, etc. On the contrary, in the shadow of the minor-radius torus nearest to the center, a preference exists for the center-falling matter, which stretches into a torus.

\section{Conclusions}

1) A dipole-toroidal vortex can be an essential element of AGN-structure, which replenishes the accretion disk.

2) In the feedback circuit, which includes twisting the vortex by radiation (or by wind) and replenishment of the accretion disk by a vortex, instability causing the bursts in active nuclei may develop.

3) Presence of centrifugal force in a vortex and lifting force connected with wind flow allow existence of a thick and cold torus.

4) The "matrjoshka" scheme of toroidal structure may explain the evolution and variability effects in AGNs.

\section{Acknowledgements}

This work is partly supported by the Ukraine President's grant GP/F8/0051.

\section{References}

Jaffe, W., Meisenheimer, K., Röttgering, H. J. A., Leinert, Ch., Richichi, A., Chesneau, O. et al. 2004, Nature 429, 47

Bliokh, K. Yu. \& Kontorovich, V. M. 2006, JETP 123, No 6, 1123 (astro-ph/0407320)

Bannikova, E. Yu. \& Kontorovich, V. M. 2006, Radio Physics \& Radio Astronomy 11, No 1, 42 (in Russian); Problems of Atomic Sci \& Techn. No 5, 146

Babadzhanyants, M. K. \& Belokon, E. T. 1985, Astrofizika 23, No 3, 459 